\title{
Complementary Barker Code excitation for Pulse-compression Thermography
}

\author{
by S. Laureti*, M. Ricci*, P. Burrascano**, D.A. Hutchins***, G.Y. Tian**** and B. Gao***** \\ * University of Calabria, Department of Informatics, Modeling, Electronics and System Engineering, Via P. Bucci \\ I-87036, Rende (CS), Italy. \{stefano.laureti,marco.ricci\}@unical.it \\ ** University of Perugia, Department of Engineering, Polo Scientifico Didattico di Terni, Strada di Pentima 4, \\ 05100, Terni (TR), Italy. pietro.burrascano@unipg.it \\ *** School of Engineering, University of Warwick, Library Road, Coventry CV4 7AL, United Kingdom. \\ D.A. Hutchins@warwick.ac.uk \\ ${ }^{* * *}$ Department of Electrical and Electronic Engineering, Newcastle University, Merz Court, NE1 7RU, Newcastle \\ upon Tyne, UK. g.y.tian@newcastle.ac.uk \\ ${ }^{* * * * * S c h o o l}$ of Automation, University of Electronic Science and Technology of China, Chengdu, Sichuan, China. \\ bingao@uestc.edu.cn
}

\section{Abstract}

In Pulse-compression thermography, the impulse response of the sample under test is retrieved by exciting the specimen via a modulated-heating stimulus, and then by pixelwise-convolving the collected thermograms via a matched filter. This paper introduces the application of a Barker Code modulating signal to Pulse-compression Thermography in a way that was not explored so far. In particular, the Barker code' features are here fully exploited by actively considering both its negative and positive bits, leading to the retrieval of two distinct thermal contributions. Those are then algebraically summed up and then convolved, leading to a faithful reconstruction of the impulse response. This novel procedure has been demonstrated on an additive manufactured PMMA sample containing artificial defects, heated-up by a single low power $50 \mathrm{~W}$ LED chip in the visible spectral range.

\section{Introduction}

Active Thermography nondestructive evaluation has been mainly exploited in two main schemes: Pulsed Thermography (PT) [1] and Lock-in Thermography (LT) [2]. In PT, the use of a short time duration yet wide frequency band heating signal maximize the feature extraction capability. Conversely, in LT the heat excitation is so that a narrower frequency band is excited but within a wider time span, thus providing less information but an increased Signal-to-Noise Ratio (SNR) with respect to PT.

In the last two decades, some other schemes have been proposed aimed at combining the advantages of both the PT and LT approaches. In this framework, Pulse Phase Thermography [3], Multi-Frequency Lock-in [4] and Pulsecompression Thermography (PuCT) [5-7] are good example of schemes aimed at maximizing the SNR while leaving unaltered the information content.

In PuCT, a coded heating stimulus is employed to heat a specimen up, and an impulse response estimate $\tilde{h}(t)$ very similar to that obtainable with a PT test - is retrieved by convolving the acquired thermograms with a proper matched filter signal. Note that here the SNR level could be increased almost arbitrarily by just extending the time duration of the coded heating stimulus. Examples of commonly employed coded signals are frequency modulated "chirp" and binary pseudo-noise codes such as the Barker code.

Pulse-compression relies on the existence of a pair of signals $\{s(t), \Psi(t)\}$ such that their convolution "*" results in a good approximation $\tilde{\delta}(t)$ of the Dirac's delta function $\delta(t)$, i.e. $s(t) * \Psi(t)=\tilde{\delta}(t) \approx \delta(t)$. Thus, an estimate $\tilde{h}(t)$ of the impulse response $h(t)$ can be retrieved by convolving pixelwise the acquired thermal signal $y(t)$ with the matched filter $\Psi(t)$ :

$$
\tilde{h}(t)=y(t) * \Psi(t)=h(t) * s(t) * \Psi(t) \approx h(t)
$$

High care should be taken to obtain an $\tilde{h}(t)$ very close to the ideal one $h(t)$, i.e. very similar to the one obtainable with a PT test with the same energy level. To this aim, Eq.(1) shows that the quantities one can play with are only (i) the coded signal $s(t)$ and (ii) the related matched filter $\Psi(t)$, which is in a first approximation the time-reversed replica of $s(t)$, i.e. $\Psi(t)=s(-t)$.

As a matter of fact, much efforts have been done in optimizing the matched filter $\Psi(t)$ for a given coded excitation signal, e.g. using window functions for tapering its amplitude, or Wiener filter approaches for shaping its frequency spectrum [8]. Moreover, different researches have shown the importance of correctly removing the step-heating $S H(t)$ contribute arising using monopolar heating source modulated by bipolar coded signals [6,8-9]

In this paper, a novel approach to optimize the PuCT procedure is introduced, which considers both the positive and negative bits of a given pseudo-noise coded excitation signal.

To gain insight into the proposed procedure, let $s(t)$ being a Barker Code $B C[n]$ of order 13 . The standard procedure to exploit the $B C[n]$ in PuCT is shown in Eq.(2):

$$
B C[n]=\{1,1,1,1,1,-1,-1,1,1,-1,1,-1,1\} \rightarrow B C[n]_{P u C T}=\{1,1,1,1,1,0,0,1,1,0,1,0,1\}
$$


meaning that each " +1 " bit is left unaltered and codes for an "on" state of the heat source, whilst each "- 1 " codes for an "off" state, i.e. no heat is provided to the specimen during the "- 1 " state. The $y(t)$ obtained by using $B[n]_{P u C T}$ is then convolved with the matched filter $\Psi(t)$ to obtain an estimate of the impulse response $\tilde{h}(t)$, see Eq.(1).

The here-presented novel approach considers the whole $B[n]$ signal as useful for the PuCT. To this aim, $B[n]$ is divided into two distinct parts, $B C_{+P u C T}$ and $B C_{-}[n]_{P u C T}$, as for Eq.(3):

$B C[n]=\{1,1,1,1,1,-1,-1,1,1,-1,1,-1,1\}=\left\{\begin{array}{l}B C_{+_{P u C T}}=\{1,1,1,1,1,0,0,1,1,0,1,0,1\} \\ B C_{-P u C T}=\{0,0,0,0,0,1,1,0,0,1,0,1,0\}\end{array}\right.$

so that the $S H(t)$ and $y(t)$ are obtained as:

$$
\begin{aligned}
& S H(t)=y_{B C_{+P u C T}}(t)+y_{B C_{-P u C T}}(t) \\
& y(t)=y_{B C_{+P u C T}}(t)-y_{B C_{-P u C T}}(t)
\end{aligned}
$$

The $y(t)$ obtained by applying Eq. $(4)$ is then convolved with $\Psi(t)=B C[-n]$ to get an impulse response estimate $\tilde{h}(t)$. Note that the proposed procedure need for two separate measurements - one using $B C_{+_{P u C T}}$ and another one with $B C_{-P U C T}$ - for being faithfully carried out.

\section{Results}

Fig.1(a-b) shows the results of the proposed procedure on a PMMA sample containing flat-bottomed holes, having their top ends located at different depth from the inspected surface. The sample was heated-up by a 50 W LED chip in the visible spectrum, and the thermograms acquired by a MWIR Xenics-ONCA camera in reflection mode at 40 FPS.

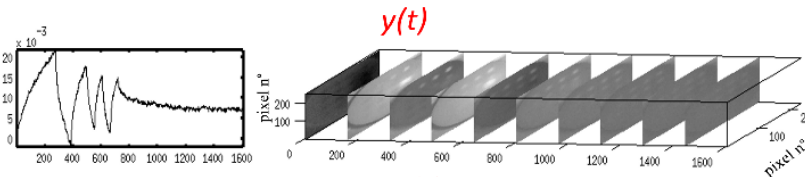

$S H(t)$

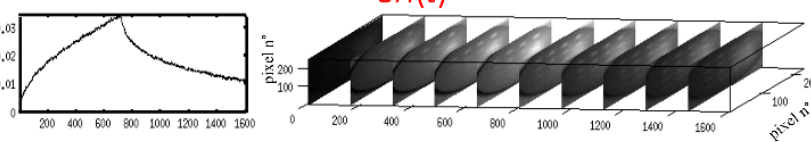

Reconstructed $h(t)$ response after PuC
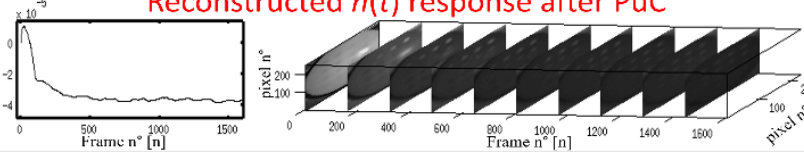
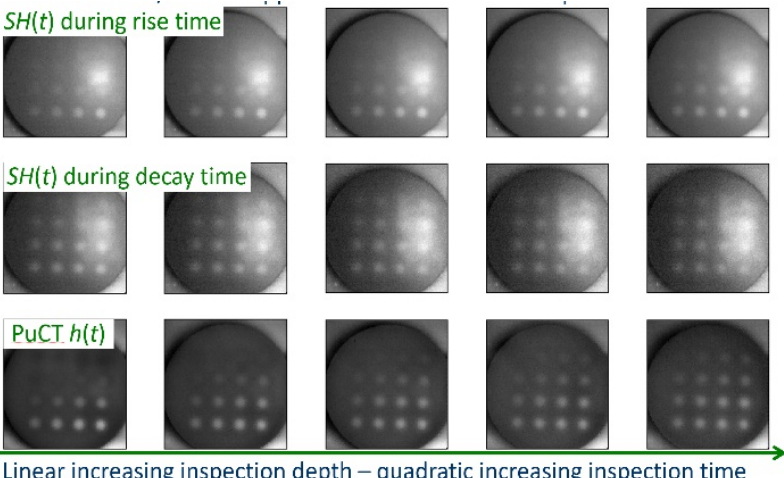

Linear increasing inspection depth - quadratic increasing inspection time

(b)

(a)

Fig. 1.(a) A single pixel trend (frame) showing the retrieved $y(t), S H(t)$ and $h(t)$ contributions, together with a series of related thermograms as time elapses; (b) a qualitative comparison among $S H(t)$ and $h(t)$ at different time instants.

\section{Conclusions}

It has been shown that the proposed novel excitation scheme can be fruitfully applied in Pulse-compression Thermography. The obtained Pulse-compression output show an increased SNR over the Step-Heating contribute and extensively overcome the outcome of previous studies on the same sample [10]. Increasingly, the need for two separate measurements is counterbalanced by the fact that a non-linear fitting procedure for removing the step-heating contribution is avoided.

\section{REFERENCES}

[1] Maldague, X. (2000). Applications of infrared thermography in nondestructive evaluation. Trends in optical nondestructive testing, 591-609.

[2] Wu, D., \& Busse, G. (1998). Lock-in thermography for nondestructive evaluation of materials. Revue générale de thermique, 37(8), 693-703.

[3] Maldague, X., \& Marinetti, S. (1996). Pulse phase infrared thermography. Journal of applied physics, 79(5), 26942698.

[4] Pitarresi, G. (2015). Lock-in signal post-processing techniques in infra-red thermography for materials structural evaluation. Experimental Mechanics, 55(4), 667-680.

[5] Mulaveesala, R., \& Tuli, S. (2005). Implementation of frequency-modulated thermal wave imaging for nondestructive sub-surface defect detection. Insight-Non-Destructive Testing and Condition Monitoring, 47(4), 206208. 
[6] Silipigni, G., Burrascano, P., Hutchins, D. A., Laureti, S., Petrucci, R., Senni, L., ... \& Ricci, M. (2017). Optimization of the pulse-compression technique applied to the infrared thermography nondestructive evaluation. NDT \& $E$ International, 87, 100-110.

[7] Yang, R., He, Y., Mandelis, A., Wang, N., Wu, X., \& Huang, S. (2018). Induction infrared thermography and thermal-wave-radar analysis for imaging inspection and diagnosis of blade composites. IEEE Transactions on Industrial Informatics, 14(12), 5637-5647.

[8] Laureti, S., Silipigni, G., Senni, L., Tomasello, R., Burrascano, P., \& Ricci, M. (2018). Comparative study between linear and non-linear frequency-modulated pulse-compression thermography. Applied optics, 57(18), D32-D39.

[9] Wu, S., Gao, B., Yang, Y., Zhu, Y., Burrascano, P., Laureti, S., ... \& Wang, Y. (2019). Halogen optical referred pulse-compression thermography for defect detection of CFRP. Infrared Physics \& Technology, 102, 103006.

[10] Malekmohammadi, H., Laureti, S., Burrascano, P., \& Ricci, M. (2019). Comparison of optimisation strategies for the improvement of depth detection capability of Pulse-Compression Thermography. Quantitative InfraRed Thermography Journal, 1-14. 